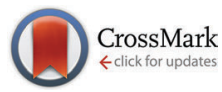

Cite this: Phys. Chem. Chem. Phys., 2017, 19, 2525

Received 29th November 2016, Accepted 21st December 2016

DOI: $10.1039 / c 6 c p 08161 d$

www.rsc.org/pccp

\title{
Molecular qubits based on potentially nuclear-spin-free nickel ions $\dagger$
}

\author{
K. Bader, ${ }^{a}$ S. H. Schlindwein, ${ }^{b}$ D. Gudat ${ }^{b}$ and J. van Slageren ${ }^{\text {ac }}$
}

Molecular qubits with the longest coherence times thus far are based on nuclear-spin-carrying central ions. These nuclear spins can cause quantum state leakage, which is detrimental to quantum algorithm performance. We present two novel molecular qubits based on potentially nuclear spin-free $\mathrm{Ni}$ in the formal oxidation state $3+.\left(\mathrm{d}_{20}-\mathrm{PPh}_{4}\right)\left[\mathrm{Ni}(\mathrm{mnt})_{2}\right]\left(\mathbf{N i}-\mathbf{m n t}, \mathrm{mnt}^{2-}=\right.$ maleonitrile-1,2-dithiolate) possesses a coherence time of up to $38.7 \mu$ s at $7 \mathrm{~K}$. Functionalization of the dithiolate ligand decreases the coherence time by a factor of only four in $\left(\mathrm{HNEt}_{3}\right)\left[\mathrm{Ni}(\mathrm{dip})_{2}\right]$ ( $\mathrm{Ni}$-dip, dip ${ }^{2-}=3$-(diphenylphosphoryl)-methylbenzene1,2-dithiolate), indicating that monoanionic Ni-dithiolene complexes are promising and robust building blocks for polynuclear molecular qubit gates.

\section{Introduction}

Speeding up computation processes and enhancing the security of encryption algorithms are major challenges in $21^{\text {st }}$ century information technology. Quantum computers have been proposed to be able to meet these challenges. The building blocks of a quantum computer inherently differ from classical bits in that they can exist in coherent superposition states. An excellent way of implementing quantum bits is the utilization of unpaired electron spins in molecular compounds (molecular qubits, MQB), for example in transition metal coordination compounds. ${ }^{1-5}$ Here the electron spin is well protected from environmental influences and at the same time the qubit's properties can be tailored by the choice of central ion, ligand and matrix of the compound. For successful application in quantum computation, the lifetime of the qubit's superposition state (coherence time) must be at least 10000 times longer than the duration of an individual quantum operation. ${ }^{6}$ For electron spin qubits, superposition states can be generated and read out by the means of pulsed EPR spectroscopy. In EPR a lower bound for the coherence time, usually called phase memory time $\left(T_{\mathrm{M}}\right)$, is obtained. The ultimate limit of the phase memory time is the characteristic time with which the molecule exchanges energy with the lattice $\left(T_{1}\right)$. Operation times of approximately $20 \mathrm{~ns}$ and phase memory times up to

\footnotetext{
${ }^{a}$ Institut für Physikalische Chemie, Universität Stuttgart, Pfaffenwaldring 55, D-70569 Stuttgart, Germany.E-mail: slageren@ipc-uni-stuttgart.de

${ }^{b}$ Institut für Anorganische Chemie, Universität Stuttgart, Pfaffenwaldring 55, D-70569 Stuttgart, Germany

${ }^{c}$ Center for Integrated Quantum Science and Technology (IQ $\left.{ }^{S T}\right)$, Stuttgart/Ulm, Germany

$\dagger$ Electronic supplementary information (ESI) available: Details on synthesis and characterization of the compounds as well as methods and additional pulsed EPR data. See DOI: 10.1039/c6cp08161d
}

$700 \mu \mathrm{s}$ lead to a figure of merit of 35000 which demonstrates that transition metal complexes are highly promising as qubits., ${ }^{3,7,8}$ Besides promising one-qubit investigations, also molecular two-qubit systems have been reported. ${ }^{9-11}$

A major challenge in implementing MQBs is currently the identification and quantification of decoherence mechanisms. In many cases the effects are poorly understood even on a qualitative level. An important example for this are nuclear spins in MQBs. Weakly coupled nuclear spins can flip randomly, leading to decoherence due to nuclear spin diffusion. An elimination of such weakly coupled nuclear spins in the vicinity of the electron therefore extends the phase memory time., ${ }^{4,12-14}$ Strong. hyperfine couplings can in turn be an asset because it provides additional spin states which can serve as further qubits or nuclear spin quantum memories. Nevertheless, strongly coupled nuclear spins can also enhance decoherence, because hard microwave pulses of 2-20 ns (with excitation bandwidths of 60-600 MHz that are comparable to many hyperfine coupling strengths) can induce spin forbidden transitions, or in other words quantum state leakage. In addition, the presence of hyperfine coupling to a nuclear spin causes the electron spins to be distributed over the available hyperfine levels, decreasing the effective number of equal MQBs in an ensemble. This has, for example, strongly deleterious effects on coupling MQBs to superconducting resonators. ${ }^{15}$ Therefore, for some applications nuclear spinfree MQB-centers are required.

Spin 1/2 paramagnetic coordination compounds with nuclear spin-free central ions can be realized with only very few transition metals. Commonly used central ions for molecular qubits such as vanadium- and copper ions have no nuclear spin-free isotopes. ${ }^{3-5}$ A nuclear-spin-free $S=1 / 2$ ion is $\operatorname{Cr}(\mathrm{v})$, but this is a highly air-sensitive. ${ }^{16}$ In contrast, $\mathrm{Ni}(\mathrm{III})$ is often air-stable, and the low natural abundance of the only magnetically active nickel 
isotope $\left(1.16 \%\right.$ for $\left.{ }^{61} \mathrm{Ni}, I=3 / 2\right)$ renders approx. $99 \%$ of all metal ions spin-free. Therefore, Ni(III) is perfectly suited for constructing truly nuclear spin-free molecular qubits. Monoanionic Ni-dithiolenes often possess strongly delocalized electronic structures, where the metal ion has $3+$ oxidation state character to some extent. ${ }^{17,18}$ Extensive investigations on these complexes and especially on $\left[\mathrm{Ni}(\mathrm{mnt})_{2}\right]^{-}\left(\mathrm{mnt}^{2-}=\right.$ maleonitrile-1,2-dithiolate $)$ have been performed because of their interesting conductivity and magnetic properties in the solid state. ${ }^{19}$ Several cw-EPR studies on monoanionic Ni-bis-dithiolene coordination compounds have been performed, including several spin-lattice relaxation measurements in concentrated single crystals. ${ }^{20-22}$ In addition, detailed ESEEM- and ENDOR-studies have been published on monoanionic Ni-bis-1,2-dithiolenes in biological systems, where it plays a crucial role in hydrogenase enzymes. ${ }^{23-25}$ Furthermore, for $\left[\mathrm{Ni}(\mathrm{mnt})_{2}\right]^{-}$salts ESEEM- and ENDOR-measurements have been performed. ${ }^{26}$ The coupling to remote nuclear spins in the ligand (e.g. the ${ }^{14} \mathrm{~N}$ nuclei in mnt) is very small $\left(A\left({ }^{14} \mathrm{~N}\right)=0.94 \mathrm{MHz}\right.$ and additionally quadrupole splitting of $3 P=2.4 \mathrm{MHz}$ ), and the electron spin is essentially in a nuclear-spin-free environment. ${ }^{18,26}$ Surprisingly, despite the variety of EPR investigations, no data on phase memory times in monoanionic Ni-bis-1,2-dithiolene compounds have been published so far.

Here we present pulsed EPR-investigations of two monoanionic Ni-bis-1,2-dithiolene (formal $\mathrm{Ni}(\mathrm{III})$ ) coordination compounds. The main objectives in this study were to detect coherence in formal Ni(III)-compounds and to identify the influences on dephasing in such new potential MQBs. For these purposes, we selected two monoanionic Ni-bis-1,2-dithiolenes (Fig. 1): $\left(\mathrm{d}_{20}-\mathrm{PPh}_{4}\right)\left[\mathrm{Ni}(\mathrm{mnt})_{2}\right]$, short Ni-mnt, where $\mathrm{mnt}^{2-}$ is maleonitrile1,2-dithiolate, and $\left(\mathrm{HNEt}_{3}\right)\left[\mathrm{Ni}(\mathrm{dip})_{2}\right]$, or Ni-dip where $\operatorname{dip}^{2-}$ is 3-(diphenylphosphoryl)-methylbenzene-1,2-dithiolate. The study of the latter compound should allow assessing the possibility to functionalize the molecular qubit (with the aim of developing two- and more qubit gates) without losing advantageous
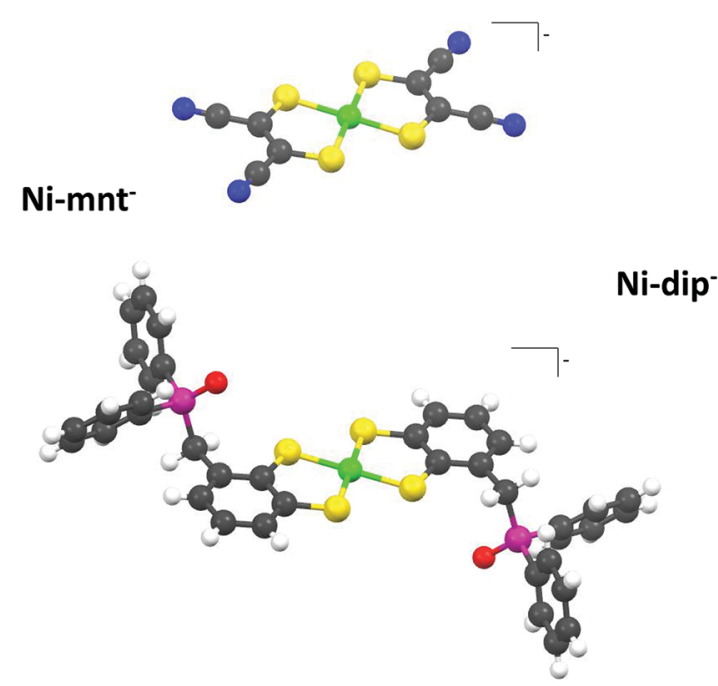

Fig. 1 Structures of investigated complex anions Ni-mnt ${ }^{-}$(top) and Ni-dip (bottom). Colors: hydrogen - white, carbon - black, nitrogen - blue, oxygen - red, phosphorous - magenta, sulfur - yellow and nickel - green. coherence properties. We demonstrate that substantial phase memory times are obtained for both compounds.

\section{Results and discussion}

The compounds were synthesized according to known procedures $^{27-30}$ and investigated by the means of pulsed Q-band EPR spectroscopy (see ESI $\dagger$ ). Both compounds are rigid square-planar bis-dithiolenes. ${ }^{30,31}$ The $\mathrm{Cu}(\mathrm{II})$ analogue of Ni-mnt was investigated as qubit previously ${ }^{4}$ and cw EPR-investigations on Ni-dip were reported recently. ${ }^{30}$ In the following we investigate the influence of ligand, matrix and measurement temperature on coherence in the first formally Ni(III) based MQBS.

Fig. 2 shows electron spin echo detected (ESE) spectra of Ni-mnt and Ni-dip in frozen solutions ( $1 \mathrm{mM}$ in $1: 1 \mathrm{~d}_{2}$-DCM/CS $\left.{ }_{2}\right)$ at $7 \mathrm{~K}$. The chosen solvent mixture displays good solubility towards the investigated compounds accompanied by a low number of nuclear-spin-carrying isotopes. The spectral line shapes for both compounds are indicative of rhombic $g$-tensors. Fits of the experimental spectra (Tables S1 and S2, ESI $\dagger$ ) were performed employing the Hamiltonian $\hat{H}=\mu_{\mathrm{B}} \mathbf{B g} \hat{\mathbf{S}}$, where $\mathbf{g}$ is the $g$-tensor, $\mu_{\mathrm{B}}$ is the Bohr magneton, $\mathbf{B}$ the external static magnetic field and $\hat{\mathbf{S}}$ the total spin vector-operator of the compound. The fits yielded the following principal $g$-tensor values for Ni-mnt: $g_{x x}=1.9935, g_{y y}=2.0404$, $g_{z z}=2.1390$ and for Ni-dip: $g_{x x}=2.0075, g_{y y}=2.0405, g_{z z}=2.1725$ (accuracy of all determined $g$-values, unless stated differently: $\Delta g= \pm 0.0005)$. In both cases, a stronger broadening ( $g$-strain) along the molecular $x$ - and $z$-axis compared to the $y$-axis was found from the simulations. The observed $g$-values are typical for square-planar monoanionic Ni-bis-dithiolenes. ${ }^{21}$

The fact that ESE detected EPR spectra can be recorded demonstrates immediately that both investigated compounds display measurable quantum coherence. The relaxation properties of Ni-mnt and Ni-dip were investigated in detail at the field position of the most intense resonance lines, indicated by the asterisks in Fig. 2. We performed inversion recovery- and Hahn echo experiments to detect $T_{1}$ and $T_{\mathrm{M}}$ respectively for both compounds at $7 \mathrm{~K}$. Fig. 3 shows the results including biexponential fit curves according to the equations $I(\tau)=I_{0}+A_{\mathrm{f}} \exp \left(-\tau / T_{1, \mathrm{f}}\right)+A_{\mathrm{s}} \exp \left(-\tau / T_{1, \mathrm{~s}}\right)$ for

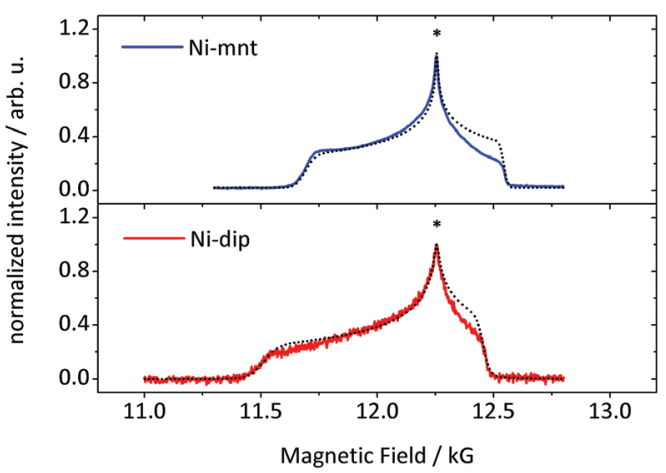

Fig. 2 ESE-detected spectra (solid lines) and simulations (dashed lines, parameters see text) of $\mathbf{N i}$-mnt (top) and $\mathbf{N i}$-dip (bottom) in $1 \mathrm{mM}$ solutions $\left(1: 1 \mathrm{~d}_{2}-\mathrm{DCM} / \mathrm{CS}_{2}\right)$ recorded at $7 \mathrm{~K}$. Asterisks mark field position for spin dynamics measurements, unless stated otherwise. 

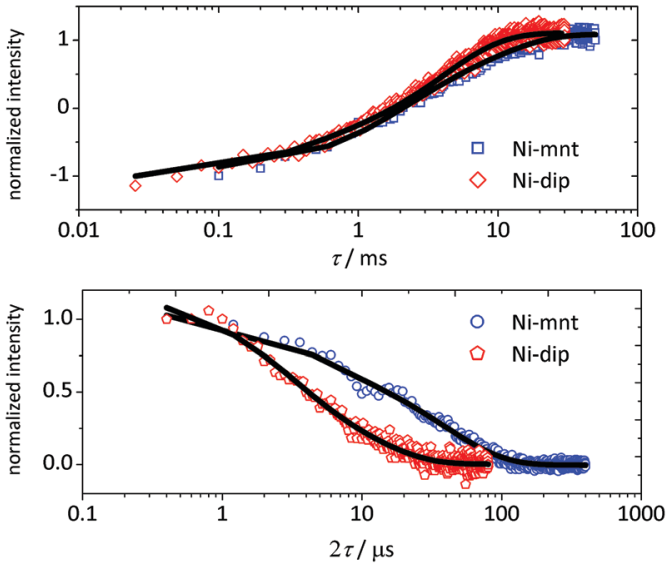

Fig. 3 Inversion Recovery (open symbols, top) and Hahn echo measurements (open symbols, bottom) for Ni-mnt and Ni-dip in $1 \mathrm{mM}$ solution $\left(1: 1 \mathrm{~d}_{2}-\mathrm{DCM} / \mathrm{CS}_{2}\right)$ recorded at $7 \mathrm{~K}$ and most intense EPR resonance line each. Solid lines correspond to biexponential fits, parameters see Table 1.

inversion recovery- and $I(2 \tau)=I_{0}+A_{\mathrm{f}} \exp \left(-2 \tau / T_{\mathrm{M}, \mathrm{f}}\right)+A_{\mathrm{s}} \exp \left(-2 \tau / T_{\mathrm{M}, \mathrm{s}}\right)$ for Hahn echo experiments. The indices $f$ and $s$ stand for fast and slow processes. Usually, the fast process is assigned to spectral diffusion and the slow process represents the actual (spin-lattice- or spin-spin-) relaxation. ${ }^{32,33} T_{1}$-times for Ni-mnt are twice as long as for Ni-dip (Table 1, Fig. S1-S4 and Tables S3, S4, ESI†). We attribute this to the rigidity of the mnt-ligand, ${ }^{4,34}$ resulting in fewer low energy vibrational modes compared to the dip-ligand. The phase memory times of both compounds are very long. The coherence time of Ni-mnt is $38.7 \pm 0.8 \mu \mathrm{s}$, which is similar to many state of the art MQBs. ${ }^{4,5,35}$ The spin-lattice relaxation- and phase memory times of Ni-mnt are not orientation dependent (variation around $10 \%$ or less, see Fig. S3, S4 and Tables S3, S4, ESI $\dagger$ ). The coherence time of Ni-dip is almost four times shorter compared to Ni-mnt. The decrease is likely due to the large number of protons in Ni-dip ( $31 \mathrm{H}$-atoms) compared to Ni-mnt (fully deuterated, $0 \mathrm{H}$-atoms). It is gratifying to see that $T_{\mathrm{M}}$ is still very long, even in this functionalized MQB. This proves that monoanionic Ni-bis-dithiolenes are versatile building blocks for more sophisticated MQB ensembles, e.g. quantum gates. In addition, in real devices the MQBs will have to be attached to a surface, e.g. by functionalization of the qubit with a linker, for selective addressability of the qubits.

In view of the longer coherence time of Ni-mnt and the availability of a diamagnetic analogue, we elected this compound

Table 1 Relaxation times extracted from biexponential fit of inversion recovery- and Hahn echo data obtained for Ni-mnt and Ni-dip in $1 \mathrm{mM}$ solutions $\left(1: 1 d_{2}-D C M / C S_{2}\right)$ at $7 \mathrm{~K}$ at the most intense resonance line. The same for deuterated and protonated doped solids at 12227 and $12209 \mathrm{G}$, respectively

\begin{tabular}{lcccc}
\hline Compound & $T_{1, \mathrm{f}} / \mathrm{ms}$ & $T_{1, \mathrm{~s}} / \mathrm{ms}$ & $T_{\mathrm{M}, \mathrm{f}} / \mu \mathrm{s}$ & $T_{\mathrm{M}, \mathrm{s}} / \mu \mathrm{s}$ \\
\hline Ni-mnt sol. & $1.6 \pm 0.2$ & $8.5 \pm 0.5$ & $3.7 \pm 0.5$ & $38.7 \pm 0.8$ \\
Ni-dip sol. & $0.33 \pm 0.05$ & $4.04 \pm 0.06$ & $2.5 \pm 0.4$ & $11 \pm 1$ \\
Ni-mnt $_{0.01 \%}$ & $0.93 \pm 0.03$ & $4.0 \pm 0.1$ & $1.109 \pm 0.004$ & $20.2 \pm 0.1$ \\
Ni-mnt $_{0.01 \%}^{\text {prot }}$ & $0.79 \pm 0.06$ & $3.8 \pm 0.1$ & & $7.78 \pm 0.03^{a}$
\end{tabular}

${ }^{a}$ Fit to stretched exponential function with stretch factor $k=1.90 \pm 0.02$. for further investigations. We probed the coherence properties in Ni-mnt $\mathbf{0}_{0.01 \%}$, which is a dilute powder of Ni-mnt in the diamagnetic matrix $\left(\mathrm{d}_{20}-\mathrm{PPh}_{4}\right)_{2}\left[\mathrm{Ni}(\mathrm{mnt})_{2}\right]$, short Ni-mnt ${ }^{\text {dia }}$. The ESE detected powder spectrum (Fig. S5 and Table S5, ESI $\dagger$ ) clearly shows two sets of three signals, attributed to two rhombic $g$-tensors. A preferential orientation along the molecular $y$-axis had to be included in the simulation to correctly reproduce the intensities. In addition, the protonated derivative of Ni-mnt $\mathbf{t}_{0.01 \%}$ shows the same pattern with nearly identical $g$-values (Fig. S10 and Table S10, ESI $\dagger$ ). These two sets of resonance lines possibly originate from two paramagnetic species with similar $g$-tensors. Similar signal doubling was previously observed for Ni-dip where the two rhombic $g$-tensors were assigned to species with slightly different local geometric structures. ${ }^{30}$ This explanation is supported by the fact that the host and dopant anions have different charges, and therefore a different number of counter ions, which will lead to local strain. ${ }^{27}$ The relaxation times for different orientations in the powder spectrum are identical within the experimental uncertainty (variation around $10 \%$ or less, see Fig. S6, S7 and Tables S6, S7, ESI $\dagger$ ), which is also found in solution.

We carried out nutation measurements on Ni-mnt ${ }_{0.01 \%}$ at the most intense resonance line of the ESE-detected spectrum. These measurements serve to demonstrate the possibility to coherently manipulate an electron spin quantum bit. Fig. 4 shows the results for different applied microwave powers on Ni-mnt ${ }_{0.01 \%}$.

Furthermore, we investigated the temperature dependence of the relaxation times of Ni-mnt ${ }_{0.01 \%}$ (Fig. 5 and Fig. S8, S9, Tables S8, S9, ESI $\dagger$ ). At all investigated temperatures (7-75 K), we find biexponential inversion recovery curves. At $7 \mathrm{~K}, T_{1} \approx 4 \mathrm{~ms}$ for the slow process of spin-lattice relaxation which accounts for approximately $50 \%$ of the measured signal. Interestingly, this is only half of the value detected for Ni-mnt in solution and appears rather small compared to a literature value of $c a .150 \mathrm{~ms}$ for tetracyanonickelate(III) (measured in a NaCl host lattice at $7 \mathrm{~K}$, Q-band). ${ }^{22}$ This suggests that the frozen solution is a more rigid environment than the doped powder. Presumably, the incorporation of Ni-mnt in the lattice of Ni-mnt results in a distorted, labile environment (see above). Going to higher temperatures,

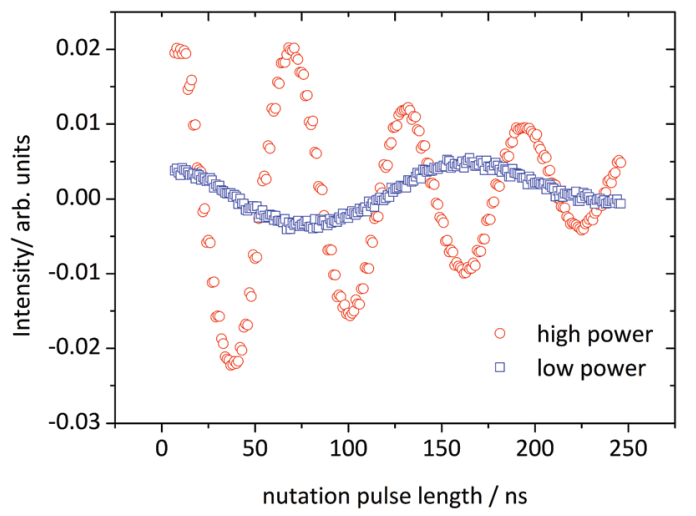

Fig. 4 Rabi oscillations of $\mathbf{N i}-\mathbf{m n t}_{0.01 \%}$, recorded at $7 \mathrm{~K}$ and most intense EPR resonance line with different powers of incident $\mathrm{MW}$-irradiation. 


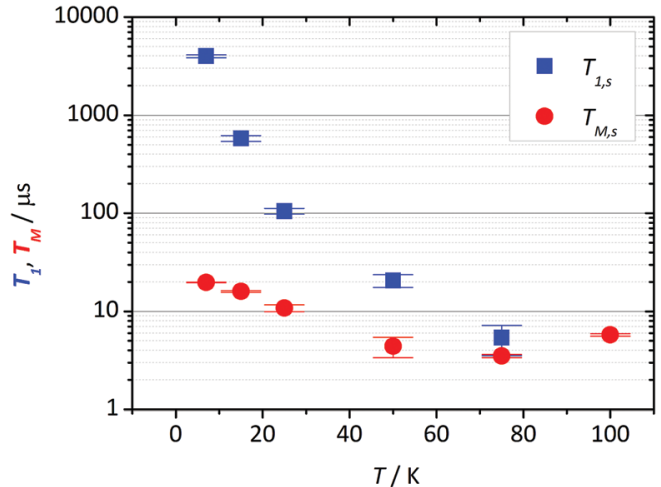

Fig. 5 Temperature dependence of relaxation times in $\mathbf{N i - m n t} \mathbf{m}_{0.01}$, recorded at most intense EPR resonance line.

both time constants of spin-lattice relaxation decrease while the importance of the slow process drops rapidly. At $75 \mathrm{~K}$ it contributes to only $c a$. $20 \%$ of the detected signal, showing a spin-lattice relaxation time of approximately $5 \mu \mathrm{s}$. At $100 \mathrm{~K}$, no signal could be observed. The temperature dependent behaviour in the protonated compound, Ni-mnt $\mathbf{t}_{0.01 \%}^{\text {prot }}$, is almost identical (Fig. S11, S12 and Table S11, ESI $\dagger$ ). The phase memory time in Ni-mnt $\mathbf{m}_{0.01 \%}$ is approximately $20 \mu$ s at $7 \mathrm{~K}$ (slow process), which is ca. 2.5 times longer than in the protonated derivative Ni-mnt ${ }_{0.01 \%}^{\mathrm{prot}}$ (Fig. S11, S13 and Table S12, ESI $\dagger$ ). This is expected because the gyromagnetic ratio of deuterium nuclei is 6.5 times smaller compared to protons, leading to less efficient nuclear spin diffusion. The factor that limits the coherence time in $\mathbf{N i - m n t} \mathbf{t}_{0.01 \%}$ is most likely the hyperfine coupling to the deuterium atoms. The phase memory time of Ni-mnt $\mathbf{m}_{0.01}$ is biexponential up to $50 \mathrm{~K}$, above that the short process vanishes in the spectrometer noise followed by the fast process at temperatures above $100 \mathrm{~K}$. At the highest measured temperature of $100 \mathrm{~K}$ - above the boiling point of nitrogen - Ni-mnt ${ }_{0.01}$ still has a phase memory time of $5.8 \mu \mathrm{s}$.

\section{Experimental}

Compounds Ni-mnt and Ni-dip were prepared by published procedures. $^{27-30}$ All measurements were performed on a homebuilt pulsed Q-band EPR spectrometer, ${ }^{36}$ with an Oxford Instruments CF935 continuous Helium flow cryostat. The samples were filled in quartz tubes, evacuated (powders) or degassed by three freeze-pump-thaw cycles (solutions) followed by flame sealing. The measurement temperature was $7 \mathrm{~K}$ unless stated otherwise. In all cases, electron spin echo detected EPR spectra were simulated with the Matlab toolbox "EasySpin". ${ }^{37}$ Unless stated differently, $g$-values were determined with an accuracy of \pm 0.0005 ; reported values of line broadenings, orientation distributions and strains are specified without error margins. Relaxation measurements were performed at field position of the most intense resonance line, marked with an asterisk in the spectra, unless stated otherwise. Spin-lattice relaxation was measured by inversion recovery- and phase memory times via Hahn echo pulse sequences; both were fitted with exponential functions (mono-, bi- or stretched exponentials). Nutation measurements were performed by applying a variable length nutation pulse followed by a Hahn echo detection scheme.

\section{Conclusions}

In conclusion, we demonstrated quantum coherence in two nickel coordination compounds for the first time. The investigated compounds Ni-mnt and Ni-dip show phase memory times of up to $38.7 \pm 0.8 \mu$ s which is comparable to state of the art MQBs. Simple coherent manipulations were performed, proving the applicability of monoanionic Ni-bis-1,2-dithiolenes as molecular qubits. The qubit properties are furthermore robust towards ligand functionalization and coherence was detected up to $100 \mathrm{~K}$. The latter may be advantageous in terms of cooling requirements if a quantum computer operating above the boiling point of nitrogen can be developed.

\section{Acknowledgements}

We thank the Institut für Anorganische Chemie (S. H. S., D. G.), the Fonds der Chemischen Industrie (K. B.) for financial support.

\section{Notes and references}

1 M. N. Leuenberger and D. Loss, Nature, 2001, 410, 789-793.

2 G. Aromi, D. Aguila, P. Gamez, F. Luis and O. Roubeau, Chem. Soc. Rev., 2012, 41, 537-546.

3 J. M. Zadrozny, J. Niklas, O. G. Poluektov and D. E. Freedman, ACS Cent. Sci., 2015, 1, 488-492.

4 K. Bader, D. Dengler, S. Lenz, B. Endeward, S.-D. Jiang, P. Neugebauer and J. van Slageren, Nat. Commun., 2014, 5, 5304.

5 L. Tesi, E. Lucaccini, I. Cimatti, M. Perfetti, M. Mannini, M. Atzori, E. Morra, M. Chiesa, A. Caneschi, L. Sorace and R. Sessoli, Chem. Sci., 2016, 7, 2074.

6 D. P. DiVincenzo, Fortschr. Phys., 2000, 48, 771-783.

7 C.-J. Yu, M. J. Graham, J. M. Zadrozny, J. Niklas, M. D. Krzyaniak, M. R. Wasielewski, O. G. Poluektov and D. E. Freedman, J. Am. Chem. Soc., 2016, 138, 14678-14685.

8 M. Atzori, L. Tesi, E. Morra, M. Chiesa, L. Sorace and R. Sessoli, J. Am. Chem. Soc., 2016, 138, 2154-2157.

9 S. Nakazawa, S. Nishida, T. Ise, T. Yoshino, N. Mori, R. D. Rahimi, K. Sato, Y. Morita, K. Toyota, D. Shiomi, M. Kitagawa, H. Hara, P. Carl, P. Höfer and T. Takui, Angew. Chem., Int. Ed., 2012, 51, 9860-9864.

10 A. Ardavan, A. M. Bowen, A. Fernandez, A. J. Fielding, D. Kaminski, F. Moro, C. A. Muryn, M. D. Wise, A. Ruggi, E. J. L. McInnes, K. Severin, G. A. Timco, C. R. Timmel, F. Tuna, G. F. S. Whitehead and R. E. P. Winpenny, npj Quantum Inf., 2015, 1, 15012.

11 D. Aguila, L. A. Barrios, V. Velasco, O. Roubeau, A. Repolles, P. J. Alonso, J. Sese, S. J. Teat, F. Luis and G. Aromi, J. Am. Chem. Soc., 2014, 136, 14215-14222. 
12 A. Ardavan, O. Rival, J. J. L. Morton, S. J. Blundell, A. M. Tyryshkin, G. A. Timco and R. E. P. Winpenny, Phys. Rev. Lett., 2007, 98, 057201.

13 C. Schlegel, J. van Slageren, G. Timco, R. E. P. Winpenny and M. Dressel, Phys. Rev. B: Condens. Matter Mater. Phys., 2011, 83, 134407.

14 C. J. Wedge, G. A. Timco, E. T. Spielberg, R. E. George, F. Tuna, S. Rigby, E. J. L. McInnes, R. E. P. Winpenny, S. J. Blundell and A. Ardavan, Phys. Rev. Lett., 2012, 108, 107204.

15 C. Bonizzoni, A. Ghirri, K. Bader, J. van Slageren, M. Perfetti, L. Sorace, Y. Lan, O. Fuhr, M. Ruben and M. Affronte, Dalton Trans., 2016, 45, 16596-16603.

16 A. Levina, L. Zhang and P. A. Lay, Inorg. Chem., 2003, 42, 767-784.

17 M. Stein, E. van Lenthe, E. J. Baerends and W. Lubitz, J. Phys. Chem. A, 2001, 105, 416-425.

18 M. Stein, Chem. - Eur. J., 2011, 17, 15046-15048.

19 N. Robertson and L. Cronin, Coord. Chem. Rev., 2002, 227, 93-127.

20 A. H. Maki, N. Edelstein, A. Davison and R. H. Holm, J. Am. Chem. Soc., 1964, 86, 4580-4587.

21 R. Kirmse, J. Stach, W. Dietzsch, G. Steimecke and E. Hoyer, Inorg. Chem., 1980, 19, 2679-2685.

22 N. V. Vugman, M. B. de Araújo, N. M. Pinhal, C. J. Magon and A. J. d. C. Filho, J. Magn. Reson., 2004, 168, 132-136.

23 A. Chapman, R. Cammack, C. E. Hatchikian, J. McCracken and J. Peisach, FEBS Lett., 1988, 242, 134-138.

24 B. Guigliarelli, C. More, A. Fournel, M. Asso, E. C. Hatchikian, R. Williams, R. Cammack and P. Bertrand, Biochemistry, 1995, 34, 4781-4790.
25 M. Saggu, C. Teutloff, M. Ludwig, M. Brecht, M.-E. Pandelia, O. Lenz, B. Friedrich, W. Lubitz, P. Hildebrandt, F. Lendzian and R. Bittl, Phys. Chem. Chem. Phys., 2010, 12, 2139-2148.

26 J. E. Huyett, S. B. Choudhury, D. M. Eichhorn, P. A. Bryngelson, M. J. Maroney and B. M. Hoffman, Inorg. Chem., 1998, 37, 1361-1367.

27 G. R. Lewis and I. Dance, J. Chem. Soc., Dalton Trans., 2000, 3176-3185.

28 L. Horner, G. Mummenthey, H. Moser and P. Beck, Chem. Ber., 1966, 99, 2782-2788.

29 W.-H. Ning, L. Zhai, J.-L. Liu, X.-M. Ren, K. Ichihashi, S. Nishihara and K. Inoue, J. Mater. Chem. C, 2015, 3, 7906-7915.

30 S. H. Schlindwein, K. Bader, C. Sibold, W. Frey, P. Neugebauer, M. Orlita, J. van Slageren and D. Gudat, Inorg. Chem., 2016, 55, 6186-6194.

31 E. I. Stiefel, Dithiolene Chemistry: Synthesis, Properties, and Applications, Progress in Inorganic Chemistry, John Wiley \& Sons, Inc., 2003, vol. 52.

32 S. Eaton and G. Eaton, in Distance Measurements in Biological Systems by EPR, ed. L. Berliner, G. Eaton and S. Eaton, Springer, US, 2002, vol. 19, pp. 29-154.

33 S. Takahashi, J. van Tol, C. C. Beedle, D. N. Hendrickson, L.-C. Brunel and M. S. Sherwin, Phys. Rev. Lett., 2009, 102, 087603.

34 M. Atzori, E. Morra, L. Tesi, A. Albino, M. Chiesa, L. Sorace and R. Sessoli, J. Am. Chem. Soc., 2016, 138, 11234-11244.

35 K. Bader, M. Winkler and J. van Slageren, Chem. Commun., 2016, 52, 3623-3626.

36 I. Tkach, A. Baldansuren, E. Kalabukhova, S. Lukin, A. Sitnikov, A. Tsvir, M. Ischenko, Y. Rosentzweig and E. Roduner, Appl. Magn. Reson., 2008, 35, 95-112.

37 S. Stoll and A. Schweiger, J. Magn. Reson., 2006, 178, 42-55. 